\title{
Construcciones híbridas de posesivo en La Pola Siero (Asturias): diferencias sociolingüísticas / Possessive hybrid constructions in La Pola Siero (Asturias): sociolinguistic differences ${ }^{1}$
}

\author{
CRISTINA BLEORȚU \\ ROMANISCHES SEMINAR \\ UNIVERSITÄT ZÜRICH
}

\begin{abstract}
RESUME: Ye intención de nueso presentar nesti trabayu les interferencies asturianes nel casu de los posesivos d'un corpus de fala semiespontánea de La Pola Siero (Asturies, norte d'España). Trátase de venticuatro informantes escoyíos acordies con tres variables pre-establecíes (sexu, edá, nivel d'estudios). Sicasí, y según se va ver nel apartáu de los resultaos, el papel más importante nel procesu de variación llingüística nun lu desempeñen les tres variables sociales sinon otres. Darréu d'ello, camiéntase que nuna situación diglósica como la de La Pola Siero, ye perimportante considerar los grupos sociales non como grupos fixos. Esto débese enforma a que, como falantes, disponemos d'unes identidaes tresportables (Zimmerman 1998) que nos permiten movenos llingüísticamente y negociar la nuesa identidá (Blommaert, Goffman 1956; Gumperz 1992; 2000; Kabatek, 1997a, 1997b, 2000, 2002, 2017).
\end{abstract}

Pallabres clave: castellanu faláu, posesivos, La Pola Siero (Asturies), llingua asturiana, sociollingüística.

ABSTRACT: In this paper, our aim is to present the Asturian interferences in the case of possessives employed in a semi-spontaneous speech corpus collected from La Pola Siero (Northern Spain). It consists of twenty-four informants who were selected according to three pre-established variables (sex, age, educational level). However, as it will be seen in the results section, the most important role in the process of linguistic variation is not played by these social variables; on the contrary, findings show that other variables are the most relevant factors. As a consequence, we believe that in a diglossic situation, as in La Pola Siero, it is very important to consider that the social groups are not definite. This is largely due to the fact that, as speakers, we have transportable identities (Zimmerman 1998) that allow us to move linguistically and to negotiate our identity (Blommaert, 2010; Goffman 1956; Gumperz 1992; Kabatek, 1997, 2000, 2002, 2017).

Keywords: spoken Spanish, possessives, La Pola Siero (Asturias), Asturian language, sociolinguistics.

1 Este trabajo ha sido financiado por FICYT (Fundación para el Fomento en Asturias de la Investigación Científica Aplicada y la Tecnología), contrato «Severo Ochoa» (referencia BP 14069). 


\section{INTRODUCCIÓN}

El objetivo principal de este trabajo es el de llevar a cabo un análisis de las interferencias y diferencias sociolingüísticas en los posesivos en el castellano hablado de La Pola Siero (norte de España) a partir de las formas castellanas y asturianas que se han documentado en un corpus de habla semiespontánea; se trata de veinticuatro encuestas cuyos informantes se seleccionaron en función de tres variables sociales preestablecidas (sexo, edad, nivel de estudios) ${ }^{2}$. Como se ha visto en el estudio de otros fenómenos (Bleorţu 2018), los rasgos asturianos se conservan con diferente grado de vitalidad según los informantes, las situaciones de comunicación y también, en cierta medida, en función de una serie de variables sociales.

La importancia lingüística del estudio de los posesivos ha sido puesta de manifiesto en numerosos trabajos lingüísticos (véase, p. ej., Álvarez Martínez 1989: 133-170; Costa Olid 1981; Fernández-Jardón Vindel 1983: 85-110, Picallo y Rigau 1999: 973-1023; etc.).

En lo que atañe a nuestra comunidad de habla, utilizando la bibliografía referente a las variedades lingüísticas de Asturias, el punto de partida para nuestro estudio han sido las monografías dialectales, que se han ido enriqueciendo a lo largo de las últimas décadas. Así pues, se han tomado en consideración, entre otros, los estudios ${ }^{3}$ sobre los posesivos en: concejo de $A$ Veiga (Fernández Vior 1997: 203-208); la zona de A Estierna [A Estierna, El Vau (Ibias), Trabáu y El Corralín (Degaña)], Fernández 1960: 173-175, donde los posesivos presentan formas acentuadas e invariables); la zona de El Valledor (Allande) (Muñiz 1978: 259-262); la comarca de El Cuarto de los Valles (Tinéu, Menéndez García 1963 [2009]: 195-198 (I), donde el lingüista advierte claramente que todas las formas encontradas son tónicas); datos del asturiano occidental (Rodríguez Castellano 1952: 180; Munthe 1988: 45); concejo de Somiedu (Cano González 2009: 110-117); concejo de Teberga (García Arias 1974: 100-101); parroquia de Santianes (Pravia) (García Valdés 1979: 94-96); concejo de Candamu (Díaz González 1986: 42-46); Oviedo [Uviéu] (parroquia de San Cloyo y términos aledaños, Martínez Álvarez 1967: 80-81); la zona del Alto Aller (1954: 208-212); concejo de Lena (Neira Martínez 1955: 49-50); concejo de Cabranes (Canellada 1944 [1996]: 24); concejo de Sobrescobiu (Conde Saiz 1978:

\footnotetext{
${ }^{2}$ Para más información sobre la metodología empleada remitimos a Bleorţu 2014 y 2015. Si en el artículo de 2014 se pensaba emplear una muestra de 5 informantes, al final se han empleado solamente 2 informantes por motivos de tiempo.

${ }^{3}$ Se organiza esta información siguiendo un orden oeste/este tanto en lo referente al territorio de Asturias como a las otras zonas del dominio ástur localizadas al sur de la Cordillera Cantábrica.
} 
147-150), parroquia de Tañes (Casu) (Armayor González 1995: 19); concejo de Casu (Calvo Aladro et al. 2000: 87-89); concejo de Parres (Vallina Alonso 1985: 101-110).

Y al sur de la cordillera: La Ribera ${ }^{4}$ (Salamanca) (Llorente Maldonado de Guevara 1947: 132-133); La Cabrera Alta, una comunidad que se sitúa en el suroeste de la provincia de León (Casado Lobato 2002: 70-71); Oseja de Sajambre, noreste de León (Fernández González 1959: 59, donde el investigador atestigua como particularidad la forma mín).

Igualmente se han tenido en cuenta datos de carácter sociolingüístico de Xixón (Prieto 1991: 21) y de Trubia y Godos (Uviéu) (Amado Rodríguez et al. 1993: 27.

Sin embargo, quizás el estudio de más interés, que conviene resaltar, es un artículo de Rodríguez-Castellano (1957: 172-187), donde se describe la producción de los posesivos en el dominio asturiano; este trabajo que se acaba de mencionar junto a manuales de gramática asturiana han sido la base de nuestro artículo.

Todos los estudios mencionados han representado un importante jalón en los estudios dialectales de Asturias, pero resultan insuficientes a la vista de las exigencias de la sociolingüística actual. Considero, por tanto, necesario estudiar los posesivos profundizando un poco más en el campo de la sociolingüística. A pesar de que no se trata más que de unas pocas pinceladas, creo poder aportar, con este análisis, un pequeño avance en este campo de estudio.

Para llevar a cabo tal tarea, me centraré a continuación en varios aspectos: (i) en primer término, se describe la situación lingüística de la comunidad que se estudia; (ii) en segundo término, la distribución de los posesivos en asturiano y castellano; (iii) en tercer término, la metodología que se ha empleado en este trabajo; (iv) en cuarto término, los resultados obtenidos; y por último (v), se presentan las conclusiones de la investigación.

\section{La Situación lingüística de La Pola Siero}

En La Pola Siero se da la situación lingüística que se puede registrar en el resto de Asturias, esto es, la de bilingüismo social diglósico (Andrés Díaz 1998, 2002a), donde conviven dos lenguas: el asturiano y el castellano. La primera es la lengua $« \mathrm{~B} »$, la variedad lingüística que se emplea en situaciones familiares e informales, mientras que la segunda es la lengua « $\mathrm{A}$ », de

\footnotetext{
${ }^{4}$ La comarca salmantina presenta características del asturiano occidental.
} 
prestigio, que se utiliza en situaciones formales. Tal situación lleva a la aparición de las interferencias en los discursos de los hablantes cuando se expresan en una de las dos lenguas, a ciertas asociaciones respecto de las dos variedades, al sentimiento de inferioridad lingüística ('language shyness') (Krashen 1998) en lo que concierne al asturiano. Como consecuencia de esta situación diglósica, en Asturias se habla de la existencia de cuatro tipos de amestaos ('mezclas') desde un punto de vista perceptivo (Andrés Díaz 2002b). En ellos aparecen interferencias, en mayor o menor grado, de las dos lenguas.

\section{LOS POSESIVOS EN ASTURIANO Y CASTELLANO}

\subsection{Los posesivos en asturiano}

En asturiano5 (GLLA 2001: 107-114; Andrés Díaz 1999: 83-88 y 2013: 373-388) las formas antepuestas son:

\begin{tabular}{|c|c|c|c|}
\hline & Persona & Masculino & Femenino \\
\hline \multirow{3}{*}{ Un poseedor } & I singular / plural & el mio $/$ los mios 6 & la mio / les mios \\
\hline & II singular / plural & el to / los tos & la to / les tos \\
\hline & III singular / plural & el so / los sos & la so / les sos \\
\hline \multirow{3}{*}{$\begin{array}{c}\text { Varios } \\
\text { poseedores }\end{array}$} & I singular / plural & $\begin{array}{c}\text { el nuesu } \sim \text { el nuestru } \\
\text { los nuesos } \sim \text { los nuestros }\end{array}$ & $\begin{array}{c}\text { la nuesa } \sim \text { la nuestra } \\
\text { les nueses } \sim \text { les nuestres }\end{array}$ \\
\hline & II singular / plural & $\begin{array}{c}\text { el vueso } \sim \text { el vuestru } \\
\text { los vuesos } \sim \text { los vuestros }\end{array}$ & $\begin{array}{c}\text { la vuesa } \sim \text { la vuestra } \\
\text { les vueses } \sim \text { les vuestres }\end{array}$ \\
\hline & III singular / plural & $\begin{array}{c}\text { el so } \\
\text { los sos }\end{array}$ & $\begin{array}{c}\text { la so } \\
\text { les sos }\end{array}$ \\
\hline
\end{tabular}

Figura 1: El paradigma de los posesivos antepuestos en el asturiano

En el caso de las formas pospuestas y nucleares existen dos modelos; no obstante, el modelo A normalmente tiene preferencia:

\footnotetext{
5 Se pueden encontrar unos breves datos sobre el tema también en la gramática de Cano González et al. (1976: 39-40).

${ }^{6}$ Esas formas se encuentran en el dialecto centro-oriental, con ciertas excepciones (p. ej. Quirós). Sin embargo, en la zona occidental sí que existe diferencia entre el masculino y el femenino, manteniéndose las formas etimológicas del posesivo (mieu miou / mia; tou / tua; sou / sua; nuesu / nuesa). Además, las formas del asturiano occidental representan una etapa evolutiva anterior a las del centro-oriental. Para más información, véase Rodríguez Castellano (1957) y Andrés Díaz (2013: 377).
} 


\begin{tabular}{|c|c|c|c|c|}
\hline & Persona & Masculino & Femenino & Neutro \\
\hline \multirow{3}{*}{$\begin{array}{c}\text { Un } \\
\text { poseedor }\end{array}$} & I singular / plural & mio / mios & mio / mios & mio / mios \\
\hline & II singular / plural & to / tos & to / tos & to / tos \\
\hline & III singular / plural & so / sos & so / sos & so / sos \\
\hline \multirow{3}{*}{$\begin{array}{c}\text { Varios } \\
\text { poseedores }\end{array}$} & I singular / plural & $\begin{aligned} \text { nuesu } & \sim \text { nuestru } \\
\text { nuesos } & \sim \text { nuestros }\end{aligned}$ & $\begin{array}{c}\text { nuesa } \sim \text { nuestra } \\
\text { nueses } \sim \text { nuestres }\end{array}$ & nueso $\sim$ nuestro \\
\hline & II singular / plural & $\begin{array}{l}\text { vueso } \sim \text { vuestru } \\
\text { vuesos } \sim \text { vuestros }\end{array}$ & $\begin{array}{c}\text { vuesa } \sim \text { vuestra } \\
\text { vueses } \sim \text { vuestres }\end{array}$ & vueso $\sim$ vuestro \\
\hline & III singular / plural & so / sos & so / sos & so / sos \\
\hline
\end{tabular}

Figura 2: El paradigma de los posesivos pospuestos en el asturiano: modelo A

\begin{tabular}{|c|c|c|c|c|}
\cline { 2 - 5 } \multicolumn{1}{c|}{} & Persona & Masculino & Femenino & Neutro \\
\hline \multirow{4}{*}{$\begin{array}{c}\text { Un } \\
\text { poseedor }\end{array}$} & I singular / plural & míu / míos & mía / míes & mío \\
\cline { 2 - 5 } & II singular / plural & tuyu / tuyos & tuya / tuyes & tuyo \\
\cline { 2 - 5 } & III singular / plural & suyu / suyos & suya / suyes & suyo \\
\hline \multirow{3}{*}{$\begin{array}{c}\text { Varios } \\
\text { poseedores }\end{array}$} & I singular / plural & $\begin{array}{c}\text { nuesu nuestru } \\
\text { nuesos nuestros }\end{array}$ & $\begin{array}{c}\text { nuesa nuestra } \\
\text { nueses nuestres }\end{array}$ & nueso nuestro \\
\cline { 2 - 6 } & II singular / plural & $\begin{array}{c}\text { vueso vuestru } \\
\text { vuesos vuestros }\end{array}$ & $\begin{array}{c}\text { vuesa vuestra } \\
\text { vueses vuestres }\end{array}$ & vueso vuestro \\
\cline { 2 - 6 } & III singular / plural & suyu / suyos & suya / suyes & suyo \\
\hline
\end{tabular}

Figura 3: El paradigma de los posesivos pospuestos en el asturiano: modelo B

A continuación, se hace necesario señalar otras particularidades de los posesivos en asturiano. Así pues, en primer lugar, se anota el uso de las formas antepuestas sin la presencia obligatoria del artículo en estos contextos:

a) Con nombres de parentesco cercano: mio pá 'mi padre'; to ma 'tu madre'; to güelu 'tu abuelo', etc.

b) Con una serie de nombres que presentan una identificación muy precisa: voi pa mio casa 'voy a mi casa'; Avisó mio suegra que nun venía 'avisó mi suegra de que no iba a venir'.

c) En tratamientos solemnes a divinidades o autoridades: So Maxestá 'Su Majestad'; Vuesa Escelencia 'Vuestra Excelencia'; 
d) En secuencias estereotipadas: nun lo dixo en so vida' 'no lo dijo en su vida';

e) En expresiones vocativas (donde no aparece nunca): Mio fiyín, ¿qué quies? 'Hijo mío, ¿qué quieres?'.

En segundo lugar, llama la atención en el asturiano hablado el uso de los sintagmas del tipo de + pronombre personal de tercera persona (él, ella, ello, ellos, elles) en vez del posesivo. Y, por último, debe mencionarse la existencia de un posesivo perifrástico la casa de vueso 'la casa de vosotros'.

\subsection{Los posesivos en castellano}

En castellano como en asturiano, los posesivos se dividen en prenominales (mi, mis, tu, tus, su, sus, cuyo, cuya, cuyos, cuyas ${ }^{8}$, nuestro, nuestra, nuestros, nuestras, vuestro, vuestra, vuestros, vuestras) у posnominales (mío, mía, míos, mías, tuyo, tuya, tuyos, tuyas, suyo, suya, suyos, suyas, nuestro, nuestra, nuеstros, nuestras, vuestro, vuestra, vuestros, vuestras).

En resumen, unas de las mayores diferencias entre las dos variedades lingüísticas es que en castellano los posesivos prenucleares son débiles, es decir, no concurren con el artículo definido, dado que conllevan en su significado el valor del artículo y son átonos; mientras que los asturianos, como los aragoneses y los gallegoportugueses, son fuertes, esto es, conllevan artículos y son tónicos.

\section{LA METODOLOGÍA EMPLEADA}

Para este estudio se ha analizado un corpus semiespontáneo que se ha recogido en el año 2014; se trata de unas 23 horas de grabación, es decir, un corpus de 215.496 palabras. Como primer paso, se han seleccionado a los informantes en función de las tres variables preestablecidas citadas arriba (sexo, edad, nivel de estudios) siguiendo, en gran parte, las directrices del proyecto PRESEEA (Proyecto para el estudio sociolingüístico del español de España y de América) ${ }^{9}$ :

\footnotetext{
${ }^{7}$ Pero también sería posible Nun lo dixo en tola so vida.

${ }^{8}$ Mencionamos el pronombre relativo porque tiene valor posesivo, que indica pertenencia a alguien mencionado previamente y actúa como adjetivo.

${ }^{9}$ PRESEEA es un proyecto coordinado por Francisco Fernández Moreno, quien se ocupa de la recogida de un corpus de español hablado representativo del mundo hispánico, donde participan alrededor de 40 equipos de investigación sociolingüística. Para más información véase: http://preseea.linguas.net/.
} 


\begin{tabular}{|c|c|c|c|c|c|c|}
\cline { 2 - 7 } \multicolumn{1}{c|}{} & \multicolumn{2}{c|}{$\begin{array}{c}\text { Generación } \\
18-37\end{array}$} & \multicolumn{2}{c|}{$\begin{array}{c}\text { Generación } \\
38-57\end{array}$} & \multicolumn{2}{c|}{$\begin{array}{c}\text { Generación } \\
>58\end{array}$} \\
\hline $\begin{array}{c}\text { Estudios } \\
\text { secundarios }\end{array}$ & $2 \mathrm{H}$ & $2 \mathrm{M}$ & $2 \mathrm{H}$ & $2 \mathrm{M}$ & $2 \mathrm{H}$ & $2 \mathrm{M}$ \\
\hline $\begin{array}{c}\text { Estudios } \\
\text { superiores }\end{array}$ & $2 \mathrm{H}$ & $2 \mathrm{M}$ & $2 \mathrm{H}$ & $2 \mathrm{M}$ & $2 \mathrm{H}$ & $2 \mathrm{M}$ \\
\hline
\end{tabular}

Figura 4: La muestra empleada

Para disponer de más información sobre los informantes, se han apuntado más datos sobre ellos; así pues, se ha tomado como modelo el esquema que utiliza Kabatek (2000) en su tesis doctoral. Es decir, nos hemos centrado en la biografía de cada individuo y se han anotado una serie de observaciones generales sobre el grado de confianza de la encuesta, los discursos recogidos, si se atestiguan o no hipercorrecciones, autocorrecciones, si el informante elide ciertas formas en sus discursos, etc. Y, por último, se ha apuntado un conjunto de datos sobre el contacto que tuvo cada hablante con las dos lenguas que conviven en La Pola Siero: dónde las aprendieron, si se identifican con ellas, en qué medida las emplean, etc.

En cuanto al análisis efectuado, ha de anotarse una precisión más: se ha evitado el uso de los posesivos en varios contextos que no se han considerado relevantes para el análisis:

a) En expresiones idiomáticas ${ }^{10}$ :

«Acuérdome una vez que acabamos en comisaría de guajes porque robamos unos caramelos en el colegio. ¡Mi madre! Unos caramelos». (Informante 5)

b) En contextos donde el informante no acaba de expresar su idea:

«Sí, sí, sí. Hombre, en La Pola, por ejemplo, hay poca gente que marche pa fuera. Por lo menos en $m i, m i$... no hay muchos que tengan capacidad económica pa poder marchar...» (Informante 2)

«Entonces también ye una manera de difundir pa les propies families del Sáhara la su historia y la $s u . .$. lo que... por lo que están luchando». (Informante 3)

c) En los contextos posnucleares; eso se debe al hecho de que las interferencias asturianas se manifiestan solamente en el caso de los posesivos prenucleares.

\footnotetext{
${ }^{10}$ Sobre este aspecto se puede consultar el estudio de Picallo y Rigau 1999: 992.
} 
d) Cuando los posesivos prenucleares van acompañados de un demostrativo o indefinido:

[T]oles mis amigues, toles mis amigues. (Informante 3)

\section{RESULTADOS}

En el castellano hablado de La Pola, aunque el posesivo conlleva muchas veces el valor del artículo, en algunas entrevistas aparece acompañado por el artículo bajo una forma híbrida (la mi hermana). Andrés Díaz (2002b: 23) ha dado algunas explicaciones sobre el comportamiento de estos posesivos. Así pues, los considera característicos de los discursos plurivarietales de los asturianos, donde aparecen elementos lingüísticos de las dos lenguas.

En nuestro rastreo de ocurrencias asturianas (híbridas en este caso), el número de ejemplos encontrados de formas prenucleares es reducido, apenas 45 ocurrencias. Así pues, el número de ejemplos hallados en nuestro corpus es muy poco elevado en comparación con los castellanos (6\%, esto es, 45 ocurrencias vs. 94\%, es decir, 678 ocurrencias):

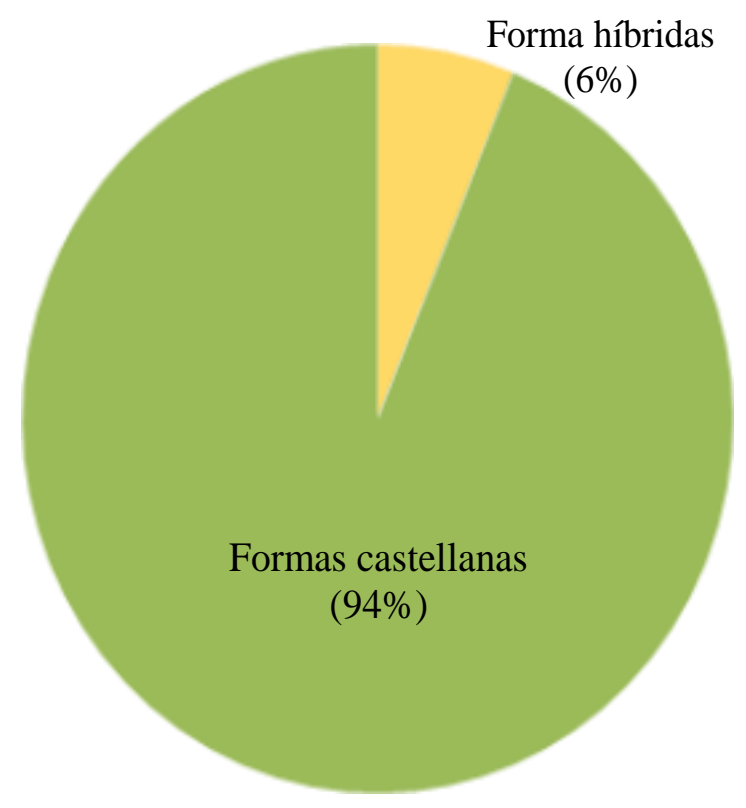

Figura 5: La dinámica de los posesivos en La Pola Siero 
Así pues, parece que los posesivos castellanos dominan sobre los asturianos en los 24 discursos recogidos, lo que nos hace recordar las palabras de Rodríguez Castellano (1957: 187): «Las formas del posesivo [...] son todavía de uso corriente en las regiones donde el dialecto se conserva con vitalidad; en las capitales de municipio y en las poblaciones importantes apenas son ya conocidas. Su olvido va íntimamente ligado a la decadencia del dialecto, cada vez más sensible».

Como consecuencia de ello, me atrevo a calificar la producción de los posesivos híbridos de esporádica y propia de un número reducido de informantes en el corpus que se ha recogido: solamente siete informantes utilizan formas prenucleares híbridas de posesivos.

Si se toman en cuenta las tres variables preestablecidas, se observa que las formas híbridas solo alcanzan a dos informantes con estudios superiores ( 6 ocurrencias). Es decir, la regla de que las formas autóctonas aparecen con más frecuencia en los informantes sin estudios se confirma, aunque no en todos los casos, pero sí está activa en una proporción superior:

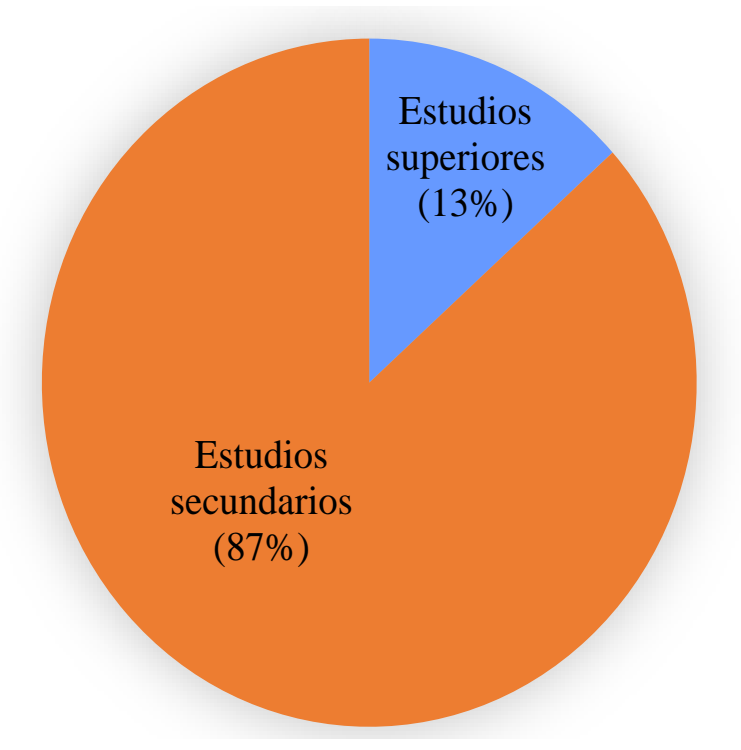

Figura 6: La dinámica de los posesivos prenucleares según el nivel de estudios

En lo que concierne a la variable sexo, la mayor proporción de formas híbridas la tienen las mujeres (25 casos, el 55,55\% del total). Así pues, podría decirse que las mujeres polesas, en los 24 discursos recogidos, emplean más posesivos híbridos. No obstante es necesaria una precisión: en 
el grupo de las mujeres se encuentra el líder lingüístico de la producción de este fenómeno; se trata de la informante 3, en cuyo discurso se atestiguan 21 ocurrencias. Si nos fijamos en su biografía lingüística se observa que se trata de una persona de lengua materna asturiana y también alfabetizada en este idioma durante su etapa escolar, variables estas que podrían influir en su discurso.

Resulta interesante, además, la variable edad, que parece ser también, en cierta medida, un factor de incidencia en la expresión de las formas híbridas. Los informantes jóvenes son los polesos que más uso hacen del fenómeno que nos ocupa (32 ocurrencias), seguidos por los de edad mediana (12 casos) y muy de lejos por los mayores (una ocurrencia):

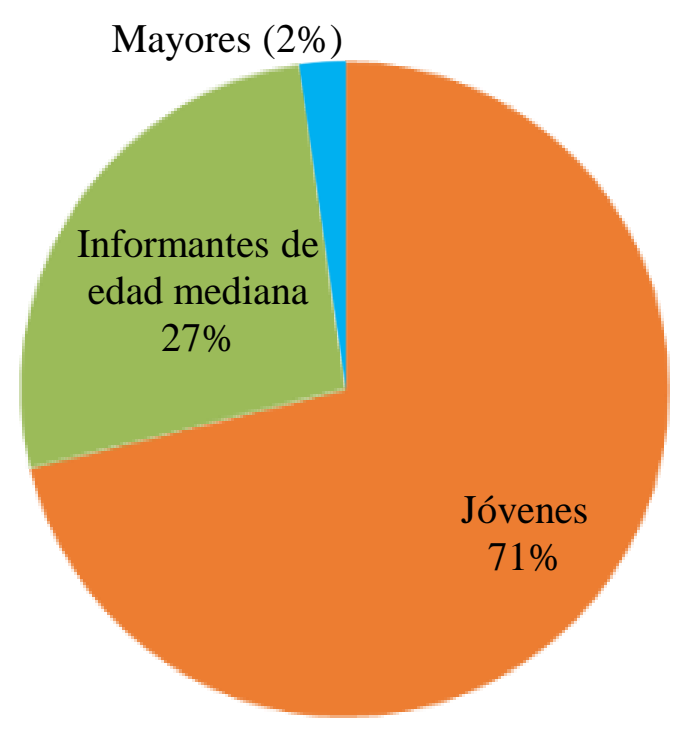

Figura 7: La dinámica de los posesivos prenucleares según la edad

Otra cuestión analizada es la difusión léxica: un uso mayor de posesivos híbridos se da con la palabra hermano y sus variaciones de género y número (once ocurrencias), seguida por amigo y sus formas. A continuación, se presenta un listado de las palabras que aparecen con un posesivo prenuclear híbrido y el número de ocurrencias documentadas en nuestro corpus: 


\begin{tabular}{|c|c|}
\hline Palabras base & Ocurrencias \\
\hline hermano & 11 \\
\hline amigo & 10 \\
\hline edad & 7 \\
\hline sobrino & 3 \\
\hline bar & 2 \\
\hline fíu & 2 \\
\hline tradiciones & 1 \\
\hline historia & 1 \\
\hline muyer & 1 \\
\hline personalidad & 1 \\
\hline marido & 1 \\
\hline vida & 1 \\
\hline cerrada & 1 \\
\hline finca & 1 \\
\hline cuñada & 1 \\
\hline llingua & \\
\hline
\end{tabular}

Figura 8: La dinámica de los posesivos prenucleares según la difusión léxica

No obstante, fijándonos en el número de ocurrencias de cada grupo social que resulta de las tres variables sociales preestablecidas (sexo, edad, nivel de estudios) se observan grandes diferencias entre los 24 informantes. Así pues, si se toma en consideración la variable sexo, se observa la producción del fenómeno solamente en el caso de cuatro informantes, en grados diferentes, en función de su identificación con la lengua local, de la identidad transportable ${ }^{11}$ que quieren adoptar. A modo de ejemplo, la informante 3 , que en su infancia ha despertado muchas veces reacciones negativas por hablar en asturiano $(\ll[\mathrm{M}]$ is propias amigas antes reíense de mí,

${ }^{11}$ Como hablantes disponemos de varias identidades lingüísticas que adoptamos en función de la situación comunicativa; de ahí la denominación de identidad transportable. 
yo tengo... yo hablo más cerrao que elles, yo pa mí la luz ta encesa, no ta prendi $(d)$ a o no sé, enllenu de... ta enllenu de cerveza, embarajar, pues así, reíense de mí porque decía eses coses... ¡Ah, mira qué mal hables! No, ye asturiano, no ye que hable mal, ye que... ye como hablan en mi casa... no ye que hable mal, ye otro idioma...») se identifica con la lengua autóctona más que las otras informantes siendo el líder lingüístico de este fenómeno.

Si se tiene en cuenta otra variable social, esto es, la edad se observa que hay tres jóvenes, tres informantes de edad mediana y uno de la tercera franja de edad que producen en sus discursos los posesivos prenucleares. Mientras que en lo que concierne al nivel de estudios, tal como se ha mencionado ya, solamente un informante con estudios superiores produce en su discurso posesivos prenucleares.

Tomando en cuenta también los resultados obtenidos en el caso de otros fenómenos morfosintácticos y léxicos (Bleorţu 2018) y siguiendo las directrices marcadas en otros estudios (Williams 1992; Kabatek 2000), creo que es mejor no considerar los grupos sociales establecidos como grupos fijos, puesto que en una situación diglósica como la de La Pola Siero hay un continuum de realizaciones, que se mueven entre los dos polos (el asturiano y el castellano), que muchas veces dependen de la situación comunicativa, de las tradiciones discursivas del discurso de cada informante, de la finalidad que persiguen los hablantes, de la identidad que quieren adoptar, etc. No solamente los resultados obtenidos en el caso de una serie larga de fenómenos lo confirman, sino también muchas veces los informantes mismos señalan su capacidad de cambiar de variedad lingüística. En palabras de uno de los veinticuatro informantes:

«Y esto lo desvirtúa, que cuando hablo con gente que no conozco no me sal el habla natural asturiana, el habla que yo hablo, que no sé lo que ye. Sin embargo, cuando hablo con gente que ye desconocida, pues me sale más hablar castellano. Yo jamás digo las escuelas. Solo me falta que cuando hablé de Les Campes haber dicho Las Campas». (Inf. 21)

\section{CONCLUSIONES}

En los apartados precedentes se han ofrecido datos acerca del uso que hacen 24 polesos de los posesivos asturianos y castellanos en el castellano hablado de La Pola Siero. Los resultados obtenidos en los análisis realizados nos permiten constatar la pervivencia de ciertas formas híbridas -que fueron atestiguadas también por Andrés Díaz (2002b), Amado Rodríguez et al. (1993), Prieto (1991), Sánchez Álvarez (1979: 262)- en grados muy diferentes y en un número reducido de sujetos. 
El sexo, la edad y el nivel de educación muestran una poca incidencia en la producción del fenómeno que nos ocupa. Así pues, puede comprobarse el predominio de las formas híbridas en los informantes con estudios secundarios, en las mujeres y en los jóvenes.

Asimismo, se ha tenido en cuenta también otra cuestión, la difusión léxica. Por consiguiente, parece que aquellas palabras que hacen referencia a la familia, a los amigos, al trabajo, es decir, secuencias textuales que exigen menor cuidado formal, son las más proclives a la aparición de los híbridos posesivos.

Los datos que acaban de ofrecerse confirman muchos de los hallazgos previos sobre otros fenómenos pero también, en esta ocasión, se advierte que la situación que se da en el caso del fenómeno que nos ocupa es un poco distinta, no se trata de un uso general como en el caso de los diminutivos, sino más bien de un fenómeno que aparece solamente en un conjunto de informantes, en los discursos que más rasgos asturianos presentan con una excepción: el informante 17, que suele ser el líder lingüístico de los demás fenómenos, no produce ninguna ocurrencia de posesivo prenuclear. Eso se puede deber al hecho de que distingue y usa bien los dos códigos lingüísticos.

También lo que se ha podido observar es que los grupos sociales no son categorías tan fijas como en la sociolingüística laboviana. Si un individuo se desvía de los usos lingüísticos de su grupo social, eso se debe a que la variación es un proceso multidimensional, donde como hablantes, a la hora de expresarnos, nos colocamos en una posición particular dentro de una matriz social compleja, sin ser una categoría de hablantes mono- o bilingües estables, sino dinámicos, armándonos con un repertorio lingüístico para expresar nuestra indexicalidad social, es decir, nuestra identidad social.

\section{Bibliografía}

ACAdemia de la Llingua Asturiana (2001): Gramática de la llingua asturiana. Uviéu, Academia de la Llingua Asturiana. ( $3^{\mathrm{a}}$ ed.).

Álvarez Martínez, M. A. (1989): El pronombre. I: Personales, Artículo, Demostrativos, Posesivos. Madrid: Arco/Libros.

AMADO RodRíGUEZ, L. et alii (1993): Transferencia llingüística nes parroquies de Trubia y Godos. Uviéu, Principáu d'Asturies.

ANDRÉS DÍAZ, R. d' (1998): «Algunas notas sobre bilingüismo y contacto de lenguas en Asturias», en A. M. ${ }^{a}$ Cano González (con la colaboración de S. Villa Basalo) (ed.): Patronymica Romanica 11. Dictionnaire historique des noms de famille romans [IX]. Actas del IX Coloquio (Oviedo, 26-29 de octubre 1995). Tübingen, Max Niemeyer Verlag: 161174.

- (1999): Gramática práctica de asturiano. Mieres, Editora del Norte. ( $3^{\mathrm{a}}$ ed.).

- (2002a): Juicios sobre la lengua asturiana. Uviéu, Ámbitu.

- (2002b): «L'asturianu mínimu urbanu. Delles hipótesis», en Lletres Asturianes 81: 21-38. 
- (2013): Gramática comparada de las lenguas ibéricas. Gijón, Trea.

Armayor GonZÁlez, H. (1995): El Habla de la Parroquia de Tanes. Córdoba, Cajasur Publicaciones.

Blas Arroyo, J. L. (2005): Sociolingüística del español. Desarrollos y perspectivas en el estudio de la lengua española en contexto social. Barcelona, Cátedra.

BLEORȚU, C. (2014): «Hacia una metodología sociolinguíística cognitiva. El estudio del habla de La La Pola Siero», en Revue Roumaine de Linguistique 4: 335-350.

- (2015): «Necesitatea unui studiu sociolingvistic în La Pola Siero», en Revista de Filoloxía Asturiana 15: 99-123.

- (2018): Aproximación al habla de La Pola Siero. Variación lingüística: descripción y percepción. Universidad de Oviedo/Universidad de Zürich. [Tesis doctoral].

Blommaert, J. (2010): The Sociolinguistics of Globalization. Cambridge, Cambridge University Press.

Calvo Aladro, C.; A. B. Martínez Prado y M. T. Cambor Portilla (2000): La fala del Conceyu Casu. Xixón, Alborá Llibros.

CAnellada, M. ${ }^{a}$ J. (1996 [1944]): El bable de Cabranes. Uviéu, Academia de la Llingua Asturiana. [Fasc. de la ed. de Madrid, Instituto «Antonio de Nebrija», 1944].

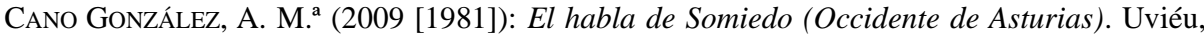
Academia de la Llingua Asturiana. [Fascs. de la ed. de la Universidade de Santiago de Compostela 1981. Separata de los n ${ }^{\text {os }} 4-\mathrm{y}$ de Verba. Anuario Galego de Filoloxía].

CANO GonZÁleZ, A. M. et alii (1976): Gramática bable. Madrid, Naranco.

Casado Lobato, M. C. (2002): El habla de la Cabrera Alta. Uviéu, Academia de la Llingua Asturiana. [Ed. fascs. de la ed. de Madrid, CSIC 1948. Anejo XLIV de la RFE].

Conde SAIZ, M. ${ }^{a}$ V. (1978): El habla de Sobrescobio. Mieres, Instituto «Bernaldo de Quirós».

Costa Olid, A. (1981): El posesivo en español. Universidad de Sevilla.

DíAz GonZÁlez, O. J. (1986): El habla de Candamo. Universidad de Oviedo.

FERnÁnDEZ, Joseph A. (1960): El habla de Sisterna. Madrid, CSIC. Anejo LXXIV de la RFE.

FERNÁNDEZ GONZÁLEZ, Ángel R. (1959): El habla y la cultura popular de Oseja de Sajambre. Oviedo: Instituto de Estudios Asturianos.

FERNÁNDEZ-JARDÓN VINDEL, J. M. (1983): Los determinantes identificadores en español: artículos, demostrativos y posesivos, Madrid, Grupodis.

FERnÁNDEZ Vior, J. A. (1997): El habla de Vegadeo (A Veiga y su concejo). Uviéu, Academia de la Llingua Asturiana. GARcía ARIAS, J. L. (1974): El habla de Teberga: sincronía y diacronía. Universidad de Oviedo. [Archivum 24, número monográfico].

García Valdés, C. C. (1979): El habla de Santianes de Pravia. Mieres, Instituto «Bernaldo de Quirós».

GOFFMAN, E. (1956): The presentation of self in everyday life. University of Edinburg/Social Sciences Research Centre.

Gumperz, J. J. (1992): «Contextualization revisited», en P. Auer y A. di Luzio (eds.), The contextualization of Language. Pragmatics and Beyond 22., Amsterdam/Philadelphia, Benjamins.

KABATEK, J. (1997a): «Dime cómo hablas y te diré quién eres», en Revista de Antropología Social 6: 215-236.

- (1997b): «Strengthening identity: differentiation and change in contemporary Galician», en J. Cheshire y D. Stein (eds.), Taming the vernacular. From Dialect to Written Standard Language London /New York, Longman:185-199.

- (2000): Os falantes como lingüistas. Tradición, innovación e interferencias no galego actual.

Vigo, Xerais.

- (2002): «Oralidad, proceso y estructura», en Pandora Oralités 2: 37-54.

- (2017): «La construcción de la historia del español a partir de los corpus: entre 'lenguas individuales' y 'tradiciones discursivas'», en M. V. Calvi, B. Hernán-Gómez Prieto y E. 
Landone (eds.), El español y su dinamismo: redes, irradiaciones y confluencias. Roma, Aispi Edizioni: 17-30.

KRASHEN, S. (1998): «Language shyness and heritage language development», en S. Krashen, L. Tse y J. McQuillan (eds.), Heritage language development. Culver, Language Education Associates: $41-49$.

Labov, W. (2006): The social Stratification of English in New York City. Cambridge/New York/Melbourne/Madrid, Cambridge University Press. ( $2^{\text {a }}$ ed.).

LloRENTE MALDONADO DE GuEVARA (1947): Estudio sobre el habla de la Ribera (comarca salmantina ribereña del Duero). Salamanca: Colegio trilingüe de la Universidad.

Martínez Álvarez, J. (1967): «Bable y Castellano en el Concejo de Oviedo». Universidad de Oviedo. [Archivum 17: 5-292].

MENÉNDEZ García 1963 [2009]: (1963): El Cuarto de los Valles, I. Oviedo: Instituto de Estudios Asturianos, [2009], Oviedo: Instituto de Estudios Asturianos.

MunTHE, A. W. (1988 [1887]: Anotaciones sobre el habla popular de una zona occidente de Asturias. Universidad de Oviedo. [Ed. fascs. de la ed. de Uppsala 1887].

MuÑIZ, C. (1978): El habla del Valledor. Estudio descriptivo del gallego asturiano de Allande (Asturias-España). Amsterdam, Academische Pers.

Neira Martínez, J. (1955): El habla de Lena. Oviedo, Instituto de Estudios Asturianos.

Picallo, M. C. y G. Rigau (1999): «El posesivo y las relaciones posesivas», en I. Bosque y V. Demonte (eds.), Gramática Descriptiva de la Lengua Española. Madrid, Espasa: 9731023.

PRIETO, C. (1991: Investigación sociollingüistica na Plaza’l Sur de Xixón. Uviéu, Serviciu de Publicaciones del Principáu d'Asturies.

Rodríguez Castellano, L. (1986 [1952]): La variedad dialectal del Alto Aller. Oviedo, RIDEA. [Facs. de la ed. de Oviedo, IDEA 1952].

- (1954): Aspectos del bable occidental. Oviedo, IDEA.

- (1957): «El posesivo en el dialecto asturiano», en BIDEA 31: 171-187.

SÁNCHEZ ÁlvareZ, M. (1979: «Consideraciones sobre el bable urbano. Notas para un estudio sociolingüístico», en Estudios y Trabayos del Seminariu de Llingua Asturiana, vol. II. Universidad de Oviedo: 259-268.

Vallina Alonso, C. (1985): El habla del sudeste de Parres. Oviedo, IDEA.

WiLLIAMS, G. (1992): Sociolinguistics: a sociological critique. Londres, Routledge.

ZimMERMAN D. H. (1998): «Discoursal identities and social identities», en C. Antaki y S. Widdicombe (eds.), Identities in talk. London, Sage: 87-10. 